Check for updates

The BMJ

Cite this as: BMJ 2021;374:n1971 http://dx.doi.org/10.1136/bmi.n1971 Published: 19 August 2021

\title{
CORONAVIRUS
}

\section{Covid-19: How many variants are there, and what do we know about them?}

\author{
Eight notable variants of SARS-CoV-2 have been found since September 2020. Elisabeth Mahase \\ reviews the line-up so far
}

Elisabeth Mahase

\section{Alpha}

Considered a variant of concern by the World Health Organization, alpha was first identified in Kent in the UK in September 2020 and drove the UK's second wave.

While it was first thought that this variant was around $70 \%$ more transmissible than the original (wild-type) SARS-CoV-2 coronavirus, data now suggest that it is $30-40 \%$ more transmissible than the original. ${ }^{1}$

Research has shown vaccine efficacy (two doses) against the alpha variant to be $74.5 \%$ with the Oxford-AstraZeneca vaccine, $93.7 \%$ with the Pfizer-BioNTech vaccine, ${ }^{2} 85.6 \%$ with the Novavax vaccine, ${ }^{3}$ and $100 \%$ with the Moderna vaccine. ${ }^{4} \mathrm{~A}$ study looking at the Sputnik V vaccine saw some reduced neutralising activity against the alpha variant, ${ }^{5}$ and Thailand's Public Health Ministry has reported that two doses of the Sinovac vaccine are $71-91 \%$ effective against alpha. ${ }^{6}$ (Video 1 )

Video 1 Covid-19: Variants of concern

\section{Beta}

First documented in South Africa in May 2020, beta is also considered a variant of concern by WHO.

The US Centers for Disease Control and Prevention (CDC) has linked beta with a 50\% increase in transmission, ${ }^{7}$ but the big worry is the emerging evidence of its ability to evade some of the existing vaccines.

Early studies indicate that the Pfizer vaccine has a slightly lower (72-75\%) effectiveness against beta than against the wild-type SARS-CoV-2, but both Pfizer and Moderna say that their vaccines are still 95\% effective against severe disease and death. Novavax (60\%) and Johnson and Johnson (57\%) fare slightly worse. And, while early studies of the Oxford-AstraZeneca vaccine seemed to show low efficacy against beta, real world data published on 23 July indicated $82 \%$ effectiveness in preventing severe disease and death from covid after a single vaccine dose. $^{8}$

Sputnik V's maker claims that it is "highly effective" against beta, but at least one study has noted a reduction in neutralising activity against this variant. ${ }^{5}$ Data on the efficacy of Sinovac's CoronaVac are lacking, although reports from Hong Kong suggested that the level of protection was $70 \%$ lower against beta than against wild-type. ${ }^{9}$

\section{Gamma}

Gamma was first identified in Manaus, Brazil, in November 2020 and is another variant of concern for WHO. At the time of writing it remains the dominant variant in South America. ${ }^{10}$

Research suggests that gamma is 1.7-2.4 times more transmissible than wild-type SARS-CoV-2. ${ }^{11}$

Few studies have been conducted to determine the efficacy of covid vaccines against the gamma variant. However, a report looking at an outbreak of gamma among employees of a goldmine in French Guiana noted a "strikingly high attack rate" among people fully vaccinated with the Pfizer vaccine, as $60 \%$ of the fully vaccinated people became infected, compared with $75 \%$ of unvaccinated miners without a history of infection. ${ }^{12}$ The manufacturers of Sputnik V claim that it is "highly effective" against variants including gamma, but a study published in July looking at antibody responses found reduced neutralising activity against gamma and other variants. ${ }^{13}$

\section{Delta}

A WHO variant of concern now dominant in Europe and the US, delta continues to drive a steep rise in cases throughout much of Asia including Bangladesh, Iran, Iraq, Japan, Kazakhstan, Malaysia, Myanmar, Pakistan, South Korea, Thailand, and Vietnam, ${ }^{14}$ as well as in India, where it was first identified in October 2020.

Delta is the most transmissible form of SARS-CoV-2 detected so far: as much as $60 \%$ more so than the alpha variant, one study estimated. Researchers have described it as an "improved" version of the alpha variant thanks to a mutation that makes it more infective in the airways. This means an increased amount of virus in the infected person such that they may expel more virus into the air, and one preprint study concluded that infected individuals had viral loads as much as 1260 times higher than people infected with wild-type SARS-CoV-2. ${ }^{15}$ Another concern is that if the delta variant is better at infecting human airway cells people may become infected after lower exposure. ${ }^{16}$

The data so far are positive regarding existing vaccines: research suggests vaccine efficacy of $67 \%$ with the Oxford-AstraZeneca vaccine and $88 \%$ with the Pfizer-BioNTech vaccine against delta, while the 
manufacturers of Sputnik V claim that it is 90\% effective against it. Another development is the emergence of delta with a ${ }_{417} \mathrm{~N}$ spike protein mutation, which has been termed delta plus. As of 23 July England had reported 45 cases of this variant. Colin Angus, a public health policy modeller and analyst, told the Washington Post that the delta plus cases had primarily been in younger people but that preliminary data showed that antibodies from vaccinated people were still effective against this variant. ${ }^{17}$

\section{Eta}

Cases of the eta variant have turned up in 72 countries including Nigeria and the UK, where it was first detected in December 2020. Little is known about eta, although the CDC said that it has the potential to reduce the neutralising ability of some monoclonal antibody treatments and convalescent plasma. WHO has declared it a "variant of interest," its second tier level of alert.

\section{lota}

As with eta, little is known about the iota variant, which was first identified in New York City, USA, in November 2020. It has so far been reported in 53 countries, and the CDC says that it has lower susceptibility to the combination bamlanivimab-etesevimab monoclonal antibody treatment. This was enough for WHO to declare it a variant of interest.

\section{Kappa}

First documented in India in October 2020, kappa is also considered a variant of interest by WHO. The CDC says that this variant may reduce the neutralisation potential of some monoclonal antibody treatments. It has been reported in 55 countries.

\section{Lambda}

First identified in Peru in December 2020, lambda became the dominant variant within three months, accounting for $80 \%$ of all cases. The swiftness and presence of mutations that could affect transmissibility and antibodies' effectiveness have made it a variant of interest for WHO. It has been detected in 41 countries but has not yet outcompeted any of the more dominant variants.

No peer reviewed studies of lambda have been conducted, but early preprint studies indicate some reduction in neutralising antibody effects from the CoronaVac (Sinovac) vaccine, ${ }^{18}$ as well as Pfizer and Moderna, although researchers say that they are confident that the latter two would remain protective. ${ }^{19}$

1 University of Oxford. Alpha variant spread via “super-seeding” event: warning over covid-19 variants. 23 Jul 2021. https://www.ox.ac.uk/news/2021-07-23-alpha-variant-spread-superseeding-event-warning-over-covid-19-variants

2 Bernal JL, Andrews N, Gower C, et al. Effectiveness of covid-19 vaccines against the B.1.617.2 (delta) variant. N Engl J Med 2021; published online 21 Jul. doi: 10.1056/NEJMoa2108891

3 Mahase E. Covid-19: Where are we on vaccines and variants?BMJ2021;372:n597. doi: 10.1136/bmj.n597 pmid: 33653708

4 Chemaitelly H, Yassine HM, Benslimane FM, et al. mRNA-1273 covid-19 vaccine effectiveness against the B.1.1.7 and B.1.351 variants and severe covid-19 disease in Qatar. Nat Med 2021; published online 9 Jul. doi: 10.1038/s41591-021-01446-y

5 Ikegame S, Siddiquey MNA, Hung CT, etal. Neutralizing activity of Sputnik V vaccine sera against SARS-CoV-2 variants. Nat Commun 2021;12:4598. doi: 10.1038/s41467-021-24909-9 pmid: 34312390

6 Sinovac jab “up to 91\% effective” after double dose. Bangkok Post 2021 Jun 29. https://www.bangkokpost.com/thailand/general/2139935/sinovac-jab-up-to-91-effective-afterdouble-dose

7 US Centers for Disease Control and Prevention. SARS-CoV-2 variant classifications and definitions. Updated Aug 2021. https://www.cdc.gov/coronavirus/2019-ncov/variants/variant-info.html

8 AstraZeneca. Vaxzevria is highly effective after one dose against severe disease or hospitalisation caused by beta and delta variants of concern. 23 Jul 2021. https://www.astrazeneca.com/mediacentre/press-releases/2021/vaxzevria-is-highly-effective-after-one-dose-against-severe-diseaseor-hospitalisation-caused-by-beta-and-delta-variants-of-concern.html
9 Cheung E. Coronavirus: Sinovac vaccine gives 70 per cent less protection against South African variant, but Hongkongers urged to still get jab. South China Morning Post 2021 Apr 20. https://sg.news.yahoo.com/coronavirus-sinovac-vaccine-gives-70-145628323.html

10 Taylor L. Covid-19: How the Brazil variant took hold of South America. BMJ2021;373:n1227. doi: 10.1136/bmi.n1227 pmid: 34016644

11 Faria NR, Mellan TA, Whittaker C, etal. Genomics and epidemiology of the P.1 SARS-CoV-2 lineage in Manaus, Brazil. Science 2021;372:815-21. . doi: 10.1126/science.abh2644 pmid: 33853970

12 Vignier N, Bérot V, Bonnave N, et al. Breakthrough infections of SARS-CoV-2 gamma variant in fully vaccinated gold miners, French Guiana, 2021. Emerg Infect Dis 2021; published online 21 Jul. doi: 10.3201/eid2710.211427

13 Gushchin VA, Dolzhikova IV, Shchetinin AM, etal. Neutralizing activity of sera from Sputnik V-vaccinated people against variants of concern (VOC: B.1.1.7, B.1.351, P.1, B.1.617.2, B.1.617.3) and Moscow endemic SARS-CoV-2 variants. Vaccines (Basel) 2021;9:779. doi: 10.3390/vaccines9070779 pmid: 34358195

14 Dyer 0. Covid-19: Indonesia becomes Asia's new pandemic epicentre as delta variant spreads. BMJ 2021;374:n1815. doi: 10.1136/bmj.n1815 pmid: 34272255

15 Li B, Deng A, Li K, et al. Viral infection and transmission in a large, well-traced outbreak caused by the SARS-CoV-2 Delta variant. medRxiv 2021.07.07.21260122 [preprint]. doi: 10.1101/2021.07.07.21260122

16 Mahase E. Delta variant: What is happening with transmission, hospital admissions, and restrictions?BMJ 2021;373:n1513. doi: 10.1136/bmj.n1513 pmid: 34130949

17 Hassan J, Beachum L. Here's what we know about the delta-plus variant. Washington Post 2021 Aug 3. https://www.washingtonpost.com/health/2021/08/03/delta-plus-coronavirus-variantexplained/

18 Acevedo ML, Alonso-Palomares L, Bustamante A, et al. Infectivity and immune escape of the new SARS-CoV-2 variant of interest lambda. medRxiv 2021.06.28.21259673 [preprint]. doi: 10.1101/2021.06.28.21259673

19 Tada T, Zhou H, Dcosta BM, Samanovic MI, Mulligan MJ, Landau NR. SARS-CoV-2 Lambda variant remains susceptible to neutralization by mRNA vaccine-elicited antibodies and convalescent serum. bioRxiv 2021.07.02.450959 [preprint]. doi: 10.1101/2021.07.02.450959

This article is made freely available for use in accordance with BMJ's website terms and conditions for the duration of the covid-19 pandemic or until otherwise determined by BMJ. You may use, download and print the article for any lawful, non-commercial purpose (including text and data mining) provided that all copyright notices and trade marks are retained. 